\title{
Mechanical properties of toothpastes with diamond abrasives
}

\author{
Hamza, Blend ; Attin, Thomas ; Paqué, Pune Nina ; Wiedemeier, Daniel B ; Wegehaupt, Florian J
}

\begin{abstract}
Objective: The aim of this study was to evaluate and compare surface roughness, gloss, and color change of dental enamel after being brushed with toothpastes containing diamond powder and traditional abrasives. Materials and methods: Seventy enamel slabs were derived from 70 bovine incisors. The slabs were brushed with six different toothpastes and artificial saliva as a negative control. The specimens were then stained with black tea mixed with citric acid ( 3 days, $\mathrm{pH}=4$ ) and again brushed with the same toothpastes. $\mathrm{Ra}$ (contact profilometer), gloss (glossmeter), and color (CIE L* $\mathrm{a}^{*} \mathrm{~b}^{*}$ system) values were measured after each step. Results: Emoform-F Diamond (contains diamond powder and traditional abrasives) offered significantly the best improvement of Ra and gloss values after the first brushing sequence and the best recovery of the brightness of enamel after staining and second brushing sequence $(\mathrm{P}<.05)$. AMC 2.5 (contains only diamond powder as abrasive) was not able to offer such improvement. Conclusion: Diamond powder as an additional abrasive in toothpastes could be able to offer a further improvement of Ra, gloss, and color values of enamel. Keywords: abrasives; diamond powder; gloss; surface roughness; toothpastes.
\end{abstract}

DOI: https://doi.org/10.1111/jerd.12588

Posted at the Zurich Open Repository and Archive, University of Zurich ZORA URL: https://doi.org/10.5167/uzh-196163

Journal Article

Accepted Version

Originally published at:

Hamza, Blend; Attin, Thomas; Paqué, Pune Nina; Wiedemeier, Daniel B; Wegehaupt, Florian J (2020). Mechanical properties of toothpastes with diamond abrasives. Journal of Esthetic and Restorative Dentistry, 32(4):416-423. DOI: https://doi.org/10.1111/jerd.12588 


\section{Mechanical properties of toothpastes with diamond abrasives}

Blend Hamza med. dent. ${ }^{1}$

Thomas Attin Prof. Dr. med. dent. ${ }^{1}$

Pune Nina Paqué Dr. med. dent. ${ }^{1}$

Daniel B. Wiedemeier PhD ${ }^{2}$

Florian J. Wegehaupt PD Dr. med. dent. ${ }^{1}$

${ }^{1}$ Clinic of Conservative and Preventive Dentistry, Center of Dental Medicine, University of Zurich, Zurich, Switzerland

${ }^{2}$ Statistical Services, Center of Dental Medicine, University of Zurich, Zurich, Switzerland

\section{Correspondence}

*Blend Hamza, Clinic of Conservative and Preventive Dentistry, Center of Dental Medicine, University of Zurich, Plattenstrasse 11, 8032 Zürich, Switzerland.

Email: blend.hamza@zzm.uzh.ch 


\section{Mechanical properties of toothpastes with diamond abrasives}

Objective: The aim of this study was to evaluate and compare surface roughness, gloss and color change of dental enamel after being brushed with toothpastes containing diamond powder and traditional abrasives.

Materials and methods: Seventy enamel slabs were derived from 70 bovine incisors. The slabs were brushed with six different toothpastes and artificial saliva as a negative control. The specimens were then stained with black tea mixed with citric acid $(3 \mathrm{~d}, \mathrm{pH}=4)$ and again brushed with the same toothpastes. $\mathrm{R}_{\mathrm{a}}$ (contact profilometer), gloss- (glossmeter) and color(CIE L*a* b* system) values were measured after each step.

Results: Emoform-F Diamond (contains diamond powder and traditional abrasives) offered significantly the best improvement of $R_{a}$ and gloss values after the first brushing sequence and best recovery of the brightness of enamel after staining and second brushing sequence $(p<0.05)$. AMC 2.5 (contains only diamond powder as abrasive) was not able to offer such improvement.

Conclusion: Diamond powder as an additional abrasive in toothpastes could be able to offer a further improvement of $R_{a^{-}}$, gloss- and color-values of enamel.

Keywords: Toothpastes, diamond powder, abrasives, surface roughness, gloss 


\section{Introduction}

The role of dental plaque in the development of caries and periodontal diseases has been well investigated and can now be considered self-evident ${ }^{1}$. Efforts should always be put in order to prohibit formation or maturation of dental plaque. Studies have shown that rough surfaces in the oral cavity - whether from tooth hard tissue or restorative/reconstructive material promote the retention, formation and maturation of dental plaque. Therefore, eliminating rough surfaces might support the prevention of caries and periodontal diseases ${ }^{2-4}$. The $R_{a}$ value is used to indicate the roughness of a surface.

Routine toothbrushing with a toothpaste is the most used method to fight dental plaque ${ }^{5}$. However, this is not the only reason that motivates people to brush their teeth. The desire to have bright and white teeth is also an important impulse ${ }^{6}$. Abrasives incorporated in toothpastes are the main ingredient responsible for removing dental plaque and extrinsic stains ${ }^{7}$. Ideally, abrasives should do its cleaning task without causing any enamel - or dentin - wear. They also should interact with the tooth surface in a way that prohibits - or at least does not promote - further accumulations of dental plaque or extrinsic stains. In other words, they should leave a smooth tooth surface behind, which makes it difficult for plaque and stains to adhere to ${ }^{8}$. Toothpastes containing diamond powder as abrasive particles were introduced to the market recently. They proclaim to be able to whiten and gently polish the teeth. The mechanical properties and effects of diamond powder has not yet been thoroughly investigated. The aim of this study was therefore to investigate the mechanical effects namely, $R_{a}$ value-, gloss- and color-change of enamel - of toothpastes containing diamond powder on dental enamel and to compare them with those of toothpastes with traditional abrasives. 
The null hypothesis of this study was that there is no difference in the change of the $R_{a}$ value, the gloss and the color of enamel when toothbrushing is performed with toothpastes utilizing only traditional abrasives (Elmex Sensitive Plus and Colgate Total) or diamond powder (Emoform-F Diamond and AMC 2.5) as abrasives.

\section{Materials and Methods}

Sample preparation

Seventy permanent bovine incisors were used for this study. A 10-mm enamel slab was milled out from the buccal surface of each incisor using a cylinder-shaped-diamond-coated trephine mill under constant water cooling. The slabs were then embedded in epoxy embedding material (Loctite Stycast, Henkel, Belgium) using a cylinder-shaped Teflon mold (Diameter = $20 \mathrm{~mm}$ ) and allowed to cure for $24 \mathrm{~h}$. Enamel slabs were then ground down in an automatic grinding machine (Struers Tegramin-30, Erkrath, Germany) using P240-Silicon-Carbide papers (Buehler, Esslingen, Germany) under constant water cooling. The grinding paper was set to rotate at 200 rotations/min, whereas the samples holder at 90 rotations/min. The grinding rotation was conducted in the same direction, with a pressure force of $5 \mathrm{~N}$ and lasted for 20 $\min$.

Baseline $R_{a}$ and gloss measurement

After being ground, baseline $R_{a}$ and gloss values were measured for all slabs. $R_{a}$ values were measured using a contact profilometer (Talysurf-50, Rank Tayler Hobson Limited, Leicester, UK). Five parallel $R_{a}$ measurements at distances of $1 \mathrm{~mm}$ were taken for each slab. Measurements started in the middle of the slab and recorded the roughness over traces of 5 
$\mathrm{mm}$ at a recording speed of $0.5 \mathrm{~mm} / \mathrm{s}$. The mean of the five measurements was then calculated and served as the $R_{a}$ baseline value - expressed in $\mu m$ - of the respected slab. Gloss measurement was performed using a glossmeter (Gloss $45^{\circ}$, Zehntner testing instruments, Sissach, Switzerland). A custom-made jig was used to fix the glossmeter measuring head and the slab always in the same position to each other. Three gloss measurements were taken for each slab. The mean value of the three measurements was calculated and served as the gloss value - expressed in gloss units (GU) - for the respective slab.

\section{First brushing sequence}

After baseline $R_{a}$ and gloss measurements, enamel slabs were randomly divided into seven groups ( $n=10$ ). Each group was brushed using a different toothpaste or formula as follows: Group 1: Artificial saliva / control group, group 2: Elmex Sensitive Plus (GABA International AG, Swidnica, Poland), group 3: Colgate Total (Colgate-Palmolive, Swidnica, Poland), group 4: AMC 2.5 basic formula (BF) - without diamond - (Fa. Microdiamant, K. Spring, Legwil, Switzerland), group 5: AMC 2.5 - with diamond -, group 6: Emoform basic formula (BF) without diamond - (Dr. Wild \& Co. AG, Lörrach, Switzerland) and group 7: Emoform-F Diamond - with diamond - . The composition of the tested toothpastes and formulas are shown in table 1.

Toothpaste slurries were freshly prepared by mixing $225 \mathrm{~g}$ of the respective toothpastes with $360 \mathrm{~g}$ of artificial saliva - after Imfeld $2010^{9}-$ and $0.45 \mathrm{~g}$ of silicon antifoam for $5 \mathrm{~min}$. The slurry was then pipetted into plastic tubes $(75 \mathrm{ml}, \mathrm{n}=10)$. Slabs were screwed tightly in a 6place-cross-brushing machine and the filled tubes were stretched tight immersing the entire slab and the first brushing sequence was started. All slabs were brushed for $25 \mathrm{~min}$ at 60 
cycles/min (totally 1500 brushing cycles). Brushing sequences were carried out using standard toothbrushes (Paro M43, Esro AG, Thalwil, Switzerland). The used toothbrushes consisted of 43 filaments. Each filament contained 36 polyamide medium-hard bristles und had a diameter of $0.2 \mathrm{~mm}$. the bristles had a stiffness of $3.7 \mathrm{~N}$. The load applied by the toothbrushes on the slabs was set at $2.5 \mathrm{~N}$ using a spring gauge.

After the first brushing sequence, slabs were rinsed with tap water and were measured again for their $R_{a}$ and gloss values. The same protocols were used here as for the baseline measurements.

\section{Baseline color measurement}

At this point, a baseline color measurement was taken for the brushed slabs. The color was measured according to the CIE L*a*b* system using a spectrophotometer (model: CM2600d, Konica Minolta Sensing, Tokyo, Japan). The spectrophotometer was set to measure a small area view (SAV) using a D65 illuminant under 100\% UV energy with an included specular component $(\mathrm{SCl})$. Prior to each measurement session, a zero and a white calibration were performed using standard backgrounds provided by the manufacturer. Three color measurements - for each $L^{*}, a^{*}$, and $b^{*}$ value - at a $10^{\circ}$ observation angle were taken for each slab. The mean value of the three measurements was calculated and served as the color value for the respective slab. The $L^{*}$ value indicates brightness (black [0] to white [100]), the $a^{*}$ value indicates chrominance (green $[-]$ to red $[+]$ ) and the $b^{*}$ value indicates chrominance (blue [-] to yellow [+]). 
Slabs staining and second brushing sequence

After measuring the baseline color, all slabs underwent a staining protocol. Each slab was stored in a plastic tube filled with $15 \mathrm{ml}$ black tea (Extra Strong, Marks \& Spencer, Chester, UK). The staining protocol lasted for three days during which the tubes were always kept in motion under a constant temperature of $37 \mathrm{C}^{\circ}$. The black tea was prepared by adding two tea bags to $380 \mathrm{ml}$ boiling water for $10 \mathrm{~min}$. Using citric acid, the $\mathrm{pH}$ of the tea was set at a value of 4 (original $\mathrm{pH}$ value of the tea was around 4.95). The black tea was prepared and changed daily. After the staining protocol, gloss and color were again measured for all stained slabs. To investigate the toothpastes' efficiency in removing stains and recovering color, the stained slabs were subjected to a second brushing sequence using the same brushing protocol mentioned above. Subsequently, gloss and color were measured again using the abovementioned protocols. Table 2 summarizes the study protocol.

\section{Statistical analysis}

The mean and standard deviation of the observed changes of the respective value $\left(\Delta R_{a}, \Delta G U\right.$, $\Delta \mathrm{L}, \Delta \mathrm{a}$ and $\Delta \mathrm{b})$ was calculated for each group at each planned observation point. The differences within the respective groups between observation points were tested using Wilcoxon-signed-rank-tests. On the other side, the differences between the groups at each observation point were tested using Kruskal Wallis tests. Whenever a significant difference $(p<0.05)$ was observed, a Benjamini-Hochberg corrected post-hoc Conover test was carried out to investigate pairwise differences between the groups. All calculations were conducted using the statistical software $\mathrm{R}^{10}$ including the packages PMCMRplus ${ }^{11}$ and multcompView ${ }^{12}$. 


\begin{tabular}{|c|c|c|}
\hline Toothpaste & Composition & Abrasive \\
\hline Elmex Sensitive Plus & $\begin{array}{l}\text { Aqua, Sorbitol, Hydroxyethylcellulose, Olaflur, } \\
\text { PEG-40, Hydrogenated castor oil, Aroma, } \\
\text { Sodium saccharin, } \mathrm{Cl} 77891\end{array}$ & Hydrated silica \\
\hline Colgate Total & $\begin{array}{l}\text { Aqua, Glycerin, PVM/MA Copolymer, Sodium } \\
\text { lauryl sulfate, Cellulose, Gum, Aroma, Sodium } \\
\text { hydroxide, Carrageenan, Sodium fluoride, } \\
\text { Triclosan, Sodium saccharin, Limonene, } \\
\text { Cl } 77891\end{array}$ & Hydrated silica \\
\hline AMC 2.5 & $\begin{array}{l}\text { Aqua, Glycerin, Sorbitol, PEG-400, Xanthan } \\
\text { gum, Texapon Z95P, Aroma, Titanium dioxide, } \\
\text { Sodium fluoride, Methylparaben, Saccharin, } \\
\text { Covarine Blue }\end{array}$ & Diamond particles \\
\hline AMC 2.5 basic formula & Similar to AMC 2.5 & None \\
\hline Emoform-F Diamond & $\begin{array}{l}\text { Aqua, Glycerin, Sorbitol, Propylene glycol, } \\
\text { Xylitol, PEG-8, PEG-40-Hydrogenated castor } \\
\text { oil, Cocamidopropyl betaine, Cellulose, Gum, } \\
\text { Potassium phosphate, Aroma, Sodium } \\
\text { chloride, Rebaudioside A, Sodiuim fluoride, } \\
\text { Limonene, Cl } 42090\end{array}$ & Silica, Diamond powder \\
\hline Emoform basic formula & Similar to Emoform-F Diamond & Silica \\
\hline
\end{tabular}

Table 1: Composition of the tested toothpastes and formulas 


\begin{tabular}{|c|c|c|c|c|c|c|c|}
\hline Samples & \multicolumn{7}{|c|}{ Bovine enamel slabs $(n=70)$} \\
\hline Grind & \multicolumn{7}{|c|}{ Silicon-Carbide papers (grit $=240$ ) } \\
\hline $\begin{array}{l}\text { Baseline } \\
\text { measurements }\end{array}$ & \multicolumn{7}{|c|}{$\begin{array}{l}\text { Gloss } \\
\mathrm{R}_{\mathrm{a}} \text { value }\end{array}$} \\
\hline Randomization & $\begin{array}{c}\text { Group } 1 \\
\text { Artificial } \\
\text { saliva } \\
\\
n=10\end{array}$ & $\begin{array}{l}\text { Group } 2 \\
\text { Elmex } \\
\text { Sensitive } \\
\text { Plus } \\
n=10\end{array}$ & $\begin{array}{c}\text { Group } 3 \\
\text { Colgate } \\
\text { Total } \\
\\
n=10\end{array}$ & $\begin{array}{l}\text { Group } 4 \\
\text { AMC } 2.5 \\
\text { (no } \\
\text { diamond) } \\
n=10\end{array}$ & $\begin{array}{c}\text { Group } 5 \\
\text { AMC } \\
2.5 \\
\\
n=10\end{array}$ & $\begin{array}{c}\text { Group } 6 \\
\text { Emoform } \\
\text { BF (no } \\
\text { diamond) } \\
n=10\end{array}$ & $\begin{array}{c}\text { Group } 7 \\
\text { Emoform-F } \\
\text { Diamond } \\
n=10\end{array}$ \\
\hline $\begin{array}{l}1^{\text {st }} \text { brushing } \\
\text { sequence }\end{array}$ & \multicolumn{7}{|c|}{$\begin{array}{l}\text { Slabs brushed with toothpaste slurries and PARO M43 toothbrushes: } \\
\qquad \text { In total } 1500 \text { cycles at a load of } 2.5 \mathrm{~N}\end{array}$} \\
\hline $1^{\text {st }}$ measurement & \multicolumn{7}{|c|}{$\begin{array}{c}\text { Gloss }(\Delta G U 1) \\
R_{a} \text { value }\left(\Delta R_{a}\right) \\
\text { color: } C L^{*} L^{*}, a^{*}, b^{*} \text { system }\end{array}$} \\
\hline Staining & \multicolumn{7}{|c|}{$3 \mathrm{~d}$ in black tea, $37 \mathrm{C}^{\circ}, \mathrm{pH}$ 4.0. Tea was changed daily } \\
\hline $2^{\text {nd }}$ measurement & \multicolumn{7}{|c|}{$\begin{array}{c}\text { Gloss }(\Delta G U 2) \\
\text { Color: } C L^{*}, a^{*}, b^{*} \text { system }(\Delta \mathrm{L} 1, \Delta a 1, \Delta b 1)\end{array}$} \\
\hline $\begin{array}{l}2^{\text {nd }} \text { brushing } \\
\text { sequence }\end{array}$ & \multicolumn{7}{|c|}{$\begin{array}{l}\text { Slabs brushed with toothpaste slurries and PARO M43 toothbrushes: } \\
\text { in total } 1500 \text { cycles at a load of } 2.5 \mathrm{~N}\end{array}$} \\
\hline $3^{\text {rd }}$ measurement & \multicolumn{7}{|c|}{$\begin{array}{c}\text { Gloss }(\Delta \mathrm{GU} 3) \\
\text { Color: } \mathrm{CIE} \mathrm{L*}, \mathrm{a}^{*}, \mathrm{~b}^{*} \text { system }(\Delta \mathrm{L} 2, \Delta \mathrm{a} 2, \Delta \mathrm{b} 2)\end{array}$} \\
\hline
\end{tabular}

Table 2: Study protocol 


\section{Results}

\section{Surface roughness $R_{a}$}

All groups showed comparable $R_{a}$ values at baseline measurement. Figure 1 demonstrates the observed change of $R_{a}$ values $\left(\Delta R_{a}\right)$ for each group after performing the first brushing sequence. Emoform-F Diamond offered significantly the best improvement - i.e. reduction of $R_{a}$ values amongst all groups $\left(\Delta R_{a}=-0.084 \mu \mathrm{m}\right)$. The second-best $R_{a}$ improvement was offered by Elmex Sensitive Plus, Colgate Total, and Emoform BF $\left(\Delta R_{a}=-0.035,-0.035\right.$ and $0.034 \mu \mathrm{m}$, respectively). AMC 2.5 offered the third-best $R_{a}$ improvement $\left(\Delta R_{a}=-0.015 \mu \mathrm{m}\right)$. This improvement was significantly less than the above-mentioned groups. Neither artificial saliva nor AMC 2.5 BF offered a significant $R_{a}$ improvement.

Gloss

All groups showed comparable gloss values at baseline measurement. Figure 2 demonstrates the recorded change of the gloss values $(\Delta G U)$ for each group at each point of observation. After performing the first brushing sequence, an improvement -i.e. increase - of gloss values was observed in all groups. Emoform-F Diamond offered significantly the best improvement of gloss amongst all groups ( $\Delta G U=18.2$ ). Emoform BF and Elmex Sensitive Plus offered the second-best improvement of gloss ( $\Delta \mathrm{GU}=4.91$ and 4.63 , respectively). The third-best gloss improvement was offered by Colgate Total $(\Delta G U=2.2)$. AMC 2.5 offered the fourth-best gloss improvement $(\Delta G U=1.3)$. This improvement was significantly less than the above-mentioned groups.

After performing the staining protocol, all groups showed a significant decrease in gloss values. Compared to the gloss values after the first brushing sequence, Emoform-F Diamond showed the highest loss of gloss $(\Delta G U=-27)$ followed by Emoform $B F(\Delta G U=-13.1)$, Colgate 
Total $(\Delta G U=-10.9)$, Elmex Sensitive Plus $(\Delta G U=-10.3), A M C 2.5(\Delta G U=-10.1), A M C 2.5 B F$ $(\Delta G U=-9)$ and artificial saliva $(\Delta G U=-6.4)$. Compared to the initial gloss values, groups brushed with Elmex Sensitive Plus and artificial saliva were less affected by the staining protocol than other groups.

After performing the second brushing sequence, gloss values were improved again for all groups. However, none of the tested toothpastes was able to recover the gloss values to the initial ones. Smaller change of gloss values was nonetheless observed in the artificial saliva group, followed by Elmex Sensitive Plus, Emoform BF, Emoform-F Diamond, AMC 2.5 BF, Colgate Total and AMC 2.5. Groups brushed with AMC 2.5 and Colgate Total showed significantly more loss of gloss values than artificial saliva group.

Color

a) $L^{*}$ values

After performing the staining protocol, $L^{*}$ values (indicate brightness (black [0] to white [100]) were decreased for all groups. Figure 3 demonstrates the change of $L^{*}$ values $(\Delta L)$ of each group at each point of observation. The group brushed with artificial saliva was significantly less affected by the staining protocol $(\Delta \mathrm{L}=-11.3)$ than all other groups except for Elmex Sensitive Plus group ( $\Delta \mathrm{L}=-18.9)$. AMC 2.5 and AMC 2.5 BF were similarly affected $(\Delta \mathrm{L}=-24.2)$. Emoform BF group was less affected than Emoform-F Diamond $(\Delta \mathrm{L}=-20.9$ and -25.5 , respectively), but the difference was not statistically significant.

After performing the second brushing sequence, $L^{*}$ values were improved in all groups. Compared to those obtained after the staining protocol, slabs brushed with Emoform- $\mathrm{F}$ Diamond showed the highest recovery of $L^{*}$ values $(\Delta \mathrm{L}=21.5)$ followed by Colgate Total $(\Delta \mathrm{L}$ 
= 10.9), Emoform BF ( $\Delta \mathrm{L}=6.9), \mathrm{AMC} 2.5(\Delta \mathrm{L}=6.4), \mathrm{AMC} 2.5 \mathrm{BF}(\Delta \mathrm{L}=5.7)$, Elmex Sensitive Plus $(\Delta \mathrm{L}=5.6)$ and artificial saliva $(\Delta \mathrm{L}=1.7)$.

Compared to the baseline values, Emoform-F Diamond group showed the smallest change in $L^{*}$ values $(\Delta L=4)$. It was followed by artificial saliva $(\Delta L=9.6)$, Elmex Sensitive Plus $(\Delta L=13.2)$, Emoform BF $(\Delta \mathrm{L}=14)$, Colgate Total $(\Delta \mathrm{L}=14.1), \mathrm{AMC} 2.5(\Delta \mathrm{L}=17.7)$ and $\mathrm{AMC} 2.5 \mathrm{BF}(\Delta \mathrm{L}=$ 18.5).

b) $a^{*}$ and $b^{*}$ values

After performing the staining protocol, both $a^{*}$ (green [-] to red [+]) and b* (blue [-] to yellow $[+]$ ) values took a boost towards positive values in all groups. Smaller change in a* and b* values was observed in the artificial saliva $(\Delta a=5.9, \Delta b=11.5)$ and Elmex Sensitive Plus $(\Delta a=$ $9.7, \Delta b=14)$ groups. Figures 4 and 5 demonstrate the observed change of $a^{*}$ and $b^{*}$ values $(\Delta a, \Delta b)$ of each group at each point of observation.

a* values were improved in all groups after the second brushing sequence. Compared to those obtained after the staining protocol, better recovery of $a^{*}$ values were observed in Colgate Total group $(\Delta \mathrm{a}=-7.2)$ followed by Emoform-F Diamond $(\Delta \mathrm{a}=-5.1)$, Emoform BF $(\Delta \mathrm{a}$ $=-4.9)$, Elmex Sensitive Plus $(\Delta \mathrm{a}=-4.2)$, AMC $2.5 \mathrm{BF}(\Delta \mathrm{a}=-3.8), \operatorname{AMC} 2.5(\Delta \mathrm{a}=-3.3)$ and artificial saliva group $(\Delta \mathrm{a}=-2.2)$. However, none of the tested toothpastes was able to recover the $a^{*}$ values to the baseline ones $(p<0.01)$. Smaller change in the $a^{*}$ values were nonetheless observed in the artificial saliva group followed by Elmex Sensitive Plus, Colgate Total, Emoform BF and Emoform-F Diamond. AMC 2.5 and AMC 2.5 BF groups showed significantly higher a* values than the artificial saliva group.

After the second brushing sequence, $b^{*}$ values were improved for all groups except for the AMC 2.5 BF group. Compared to those obtained after the staining protocol, better recovery 
of $b$ * values were observed in Colgate Total group $(\Delta b=-5.6)$ followed by Emoform BF $(\Delta b=$ -3.6), artificial saliva $(\Delta b=-2.6)$, Emoform-F Diamond $(\Delta b=-2.5)$, Elmex Sensitive Plus $(\Delta b=-$ 2.5), AMC 2.5 $(\Delta b=-1.6)$. AMC 2.5 BF caused a worsening of $b^{*}$ values $(\Delta b=+0.4)$. None of the tested toothpastes was able to recover the $b^{*}$ values to the baseline ones $(p<0.01)$. Smaller change in the $b^{*}$ values were nonetheless observed in the artificial saliva group followed by Elmex Sensitive Plus, Colgate Total, Emoform BF, AMC 2.5, Emoform-F Diamond and AMC $2.5 \mathrm{BF}$.

\section{Discussion}

People brush their teeth because of biological and social reasons. While the biological reasons include obtaining healthy gums and teeth, the social reasons include obtaining shiny white teeth and fresh breath ${ }^{5}$. The macroscopic and microscopic surface properties of the tooth surface play an important role in enhancing bacterial or staining accumulation ${ }^{13}$. They also determine how bright a tooth appears ${ }^{14}$. Due to their daily interaction with teeth surfaces, toothpastes affect the above-mentioned properties. This study was thus carried out to investigate how toothpastes change the microscopic surface properties of the tooth surfaces and to compare those changes resulting from toothpastes with diamond powder to those resulting from toothpastes with traditional abrasives.

Bovine enamel was used in this study. Kwon et al. ${ }^{15}$ indicated a difference in absolute reflectance values - which then were calculated to $L^{*} a * b *$ values - between human and bovine enamel. Higher $L^{*}$ values were measured in bovine enamel. The authors attributed that to differences in diet and to the relatively younger age at which the bovine were sacrificed. On the other hand, bovine enamel shares similar chemical and physical properties with human enamel which make it a suitable alternative ${ }^{16}$. Moreover, they have larger 
surfaces, could easily be obtained and have already been used in several surface-roughness and appearance-related studies ${ }^{8,17,18}$. Further studies comparing the susceptibility to glossand color-change between human and bovine enamel might be advisable. However, the values in this study were calculated under standardized conditions and are therefore relative to each other and should be able to be compared.

First brushing sequence ( $R_{a}$ and gloss)

The measurement of surface roughness is an important aspect of the surface property. $R_{a}$ value is internationally accepted as the parameter of surface roughness ${ }^{19}$. Mechanical profilometry is one of the most accepted and widely used techniques to assess surface roughness ${ }^{20}$. All slabs were firstly ground using P240-Silicon-Carbide papers. This grinding results in a mean $R_{a}$ value of $0.35 \pm 0.06 \mu \mathrm{m}$ which is in the range of the surface roughness found for sound human enamel $(0.12 \pm 0.03 \mu \mathrm{m} \text { to } 0.59 \pm 0.11 \mu \mathrm{m})^{21,22}$.

Gloss is a feature of visual appearance. It originates from the geometrical distribution of a light reflected from a surface, and consequently plays an important role in esthetic appearance ${ }^{14}$.

Both $\mathrm{R}_{\mathrm{a}}$ and gloss values were best improved in Emoform-F Diamond group. The significant better improvement of $R_{a}$ and gloss values offered by Emoform-F Diamond compared to Emoform BF can only be contributed to the addition of diamond powder. Even though AMC 2.5 also offered a significant improvement of both values, it was the least effective compared to all groups and did not significantly improve the $R_{a}$ or gloss values compared to AMC $2.5 \mathrm{BF}$. The considerable difference of $R_{a}$ and gloss improvement between Emoform-F Diamond and AMC 2.5 could be attributed to the fact that the last has only diamond powder as abrasive, while Emoform-F Diamond also contains traditional abrasives beside diamond powder. Other 
factors that could lead to such difference include volume percentage of the abrasives inside each toothpaste and their geometrical properties. It should be noted that none of the toothpastes were tested for their abrasion effect in this study. However, Tawakoli et al. ${ }^{23}$ found toothpastes with diamond abrasives to have a low abrasivity towards dentin and rather high abrasivity towards enamel compared to other commercially available toothpastes. In another study, Elmex Sensitive Plus was found to have a low abrasivity towards dentin (RDA $=26)$, whereas Colgate Total had a strong abrasivity $(\operatorname{RDA}=120)^{24}$. The above-mentioned benefits should always be balanced with the potential abrasivity toothpastes could cause to enamel and dentin.

\section{Staining protocol (gloss and color)}

In this study, black tea mixed with citric acid was used as staining solution. Tea has shown a high capacity to stain teeth and has been used as a staining solution in many studies ${ }^{25-27}$. The addition of citric acid until a $\mathrm{pH}$ value of 4 is reached aimed to obtain a standardized staining solution to be used on all slabs. Under such $\mathrm{pH}$ value and temperature $\left(37 \mathrm{C}^{\circ}\right)$ and since tea is undersaturated in regard of calcium and phosphate, it could be anticipated that enamel slabs underwent an erosive attack during the staining protocol ${ }^{28}$. Slabs were stained continuously for 3 days. This duration is exaggerated and does not represent the much shorter time teeth get in contact with tea while drinking. It was nevertheless chosen to obtain a strong staining and then to evaluate the effectiveness of the tested toothpastes in recovering gloss and color under such extreme situation. Color was measured using the threedimensional color space CIE-Lab. This system allows an objective determination of color and has been used in various studies to evaluate whitening effect of bleaching products $8,18,27,29$. 
Prior to the staining protocol, slabs brushed with Emoform-F Diamond showed significantly smoother surfaces and better gloss than slabs brushed with artificial saliva or Elmex Sensitive Plus. It might thus be expected that the much smoother enamel slabs should absorb less color than rougher ones. This was, however, not the case in this study. As shown in Figures 2 to 5 , gloss ( $\Delta \mathrm{GU} 2)$ and color $(\Delta \mathrm{L} 1, \Delta \mathrm{a} 1$ and $\Delta \mathrm{b} 1)$ were significantly less affected by the staining protocol in the artificial saliva group compared to the Emoform-F Diamond group. This was also true for the slabs brushed with Elmex Sensitive Plus which were also less affected by the staining protocol than Emoform-F Diamond. As mentioned above, slabs were simultaneously eroded while being stained. Therefore, it is plausible to attribute the differences of $\Delta G U 1$, $\Delta \mathrm{L} 1, \Delta \mathrm{a} 1$ and $\Delta \mathrm{b} 1$ between the groups to a possible protection against erosion offered in the less-affected groups. However, Batista et al. ${ }^{30}$ excluded such an erosion-protective effect for various formulas of artificial saliva as those are not able to build a protective pellicle layer on the enamel. On the other hand, Baumann et al. ${ }^{31}$ found artificial saliva to have a certain erosion-protective effect. They attributed this protective effect to the presence of sodium and chloride ions which suppress the dissolution of hydroxyapatite. It should be mentioned, that the erosive attack was undertaken differently in each of the above-mentioned studies (hydrochloric acid pH $2.6 / 1 \mathrm{~min}^{30}$ and citric acid pH $3.6 / 1 \mathrm{~min}^{31}$ ). Eventually, it remains unclear whether the artificial saliva used in this study could after all offer some kind of erosion protection and thus alter the susceptibility to color adsorption. The better values observed in Elmex Sensitive Plus group could also be connected to a possible protection offered against erosion. 


\section{Second brushing sequence (gloss and color)}

After the second brushing sequence gloss $(\Delta \mathrm{GU} 3)$ and color $(\Delta \mathrm{L} 2, \Delta \mathrm{a} 2$ and $\Delta \mathrm{b} 2)$ values were generally improved in all groups, but not totally recovered to baseline values. This might be contributed to the excessively long staining duration ( $3 \mathrm{~d})$, although, the brushing time of 25 min was also rather excessive, not representing the actual brushing time of an individual.

Differences between the groups with regard to $\Delta \mathrm{GU} 1$ were much higher with regard to $\Delta \mathrm{GU} 3$ (see figure 2). This is especially interesting for Emoform-F Diamond and Emoform BF. After the second brushing sequence, it seems to be fair to expect that $\Delta G U 3$ values of Emoform- $F$ Diamond would be better recovered than Emoform BF, which was not the case. However, Emoform-F Diamond could significantly better recover $\Delta \mathrm{L}$ values compared to its basic formula and to all other groups as well. In other words, the addition of diamond particles supported the recovery of the brightness of enamel without having distinct effect on recovering gloss. It is worth to mention again that the slabs were excessively stained once and were excessively brushed once with the tested toothpastes. A better recovery of color and gloss might have been observed if staining and brushing were carried out intermittently for shorter times which would have better addressed the every-day situation.

The null hypothesis of this study has to be rejected for Emoform-F Diamond group and could not be rejected for AMC 2.5 group. Emoform-F Diamond was able to improve $R_{a}$, gloss and color values compared to toothpastes with traditional abrasives, Whereas AMC 2.5 - also containing diamond particles - was not able to offer such improvement. Different volume percentage and geometrical properties of diamond particles as well as the presence or absence of traditional abrasives in toothpastes could be responsible for such differences ${ }^{23}$. 


\section{Conclusion}

Within the limits of this study it could be concluded that the addition of diamond powder to toothpastes could offer a further reduction of surface roughness and optimize the gloss and brightness of enamel as compared to only traditional abrasives. 


\section{References}

1. Colombo APV, Tanner ACR. The role of bacterial biofilms in dental caries and periodontal and peri-implant diseases: A historical perspective. J Dent Res. 2019;98(4):373-385.

2. Quirynen $\mathrm{M}$, Bollen $\mathrm{CM}$. The influence of surface roughness and surface-free energy on supra- and subgingival plaque formation in man. A review of the literature. $J$ Clin Periodontol. 1995;22(1):1-14.

3. Anami LC, Pereira CA, Guerra E, Assunção e Souza RO, Jorge AO, Bottino MA. Morphology and bacterial colonisation of tooth/ceramic restoration interface after different cement excess removal techniques. J Dent. 2012;40(9):742-749.

4. Bollen CM, Lambrechts $P$, Quirynen M. Comparison of surface roughness of oral hard materials to the threshold surface roughness for bacterial plaque retention: a review of the literature. Dent Mater. 1997;13(4):258-269.

5. Valkenburg C, Else Slot D, Van der Weijden GF. What is the effect of active ingredients in dentifrice on inhibiting the regrowth of overnight plaque? A systematic review. Int J Dent Hyg. 2019.

6. Graça SR, Albuquerque TS, Luis HS, et al. Oral Health Knowledge, Perceptions, and Habits of Adolescents from Portugal, Romania, and Sweden: A Comparative Study. J Int Soc Prev Community Dent. 2019;9(5):470-480.

7. Epple M, Meyer F, Enax J. A Critical Review of Modern Concepts for Teeth Whitening. Dent J (Basel). 2019;7(3).

8. Silva EMD, Maia JNDS, Mitraud CG, Russo JDES, Poskus LT, Guimarães JGA. Can whitening toothpastes maintain the optical stability of enamel over time? J Appl Oral Sci. 2018;26:e20160460.

9. Imfeld T. Standard operation procedures for the relative dentin abrasion : (RDA) method used at Zurich University. In. Vol 21: J Clin Dent; 2010:S11-S12.

10. R Core Team. R: A language and environment for statistical computing. R Foundation for Statistical Computing, Vienna, Austria. 2015; https://www.R-project.org/.

11. Pohlert T. PMCMRplus: Calculate Pairwise Multiple Comparisons of Mean Rank Sums Extended. R package version 1.4.0. 2018; https://CRAN.Rproject.org/package=PMCMRplus.

12. Graves S, Piepho HP, Selzer L, et al. multcompView: Visualizations of Paired Comparisons. R package version 0.1-7. 2015; https://CRAN.Rproject.org/package $=$ multcompView.

13. Park JW, An JS, Lim WH, Lim BS, Ahn SJ. Microbial changes in biofilms on composite resins with different surface roughness: An in vitro study with a multispecies biofilm model. J Prosthet Dent. 2019.

14. Obein G, Knoblauch K, Viénot F. Difference scaling of gloss: nonlinearity, binocularity, and constancy. J Vis. 2004;4(9):711-720.

15. Kwon YH, Huo MS, Kim KH, Kim SK, Kim YJ. Effects of hydrogen peroxide on the light reflectance and morphology of bovine enamel. J Oral Rehabil. 2002;29(5):473-477.

16. Arango-Santander S, Montoya C, Pelaez-Vargas A, Ossa EA. Chemical, structural and mechanical characterization of bovine enamel. Arch Oral Biol. 2019;109:104573.

17. Vieira-Junior WF, Lima DA, Tabchoury CP, Ambrosano GM, Aguiar FH, Lovadino JR. Effect of toothpaste application prior to dental bleaching on whitening effectiveness and enamel properties. Oper Dent. 2016;41(1):E29-38. 
18. Wiegand A, Vollmer D, Foitzik M, Attin R, Attin T. Efficacy of different whitening modalities on bovine enamel and dentin. Clin Oral Investig. 2005;9(2):91-97.

19. Cazzaniga G, Ottobelli M, Ionescu A, Garcia-Godoy F, Brambilla E. Surface properties of resin-based composite materials and biofilm formation: A review of the current literature. Am J Dent. 2015;28(6):311-320.

20. O'Donnell KA. Effects of finite stylus width in surface contact profilometry. Appl Opt. 1993;32(25):4922-4928.

21. Kodaka T, Kobori M, Hirayama A, Abe M. Abrasion of human enamel by brushing with a commercial dentifrice containing hydroxyapatite crystals in vitro. J Electron Microsc (Tokyo). 1999;48(2):167-172.

22. Erdur EA, Akın M, Cime L, ileri Z. Evaluation of enamel surface roughness after various finishing techniques for debonding of orthodontic brackets. Turk J Orthod. 2016;29(1):1-5.

23. Tawakoli PN, Becker K, Attin T. Abrasive effects of diamond dentifrices on dentine and enamel. Swiss Dent J. 2018;128(1):14-19.

24. Tawakoli PN, Sener B, Attin T. Mechanical effects of different Swiss market-leading dentifrices on dentin. Swiss Dent J. 2015;125(11):1210-1219.

25. Attin T, Manolakis A, Buchalla W, Hannig C. Influence of tea on intrinsic colour of previously bleached enamel. J Oral Rehabil. 2003;30(5):488-494.

26. Yetkiner E, Wegehaupt F, Wiegand A, Attin R, Attin T. Colour improvement and stability of white spot lesions following infiltration, micro-abrasion, or fluoride treatments in vitro. Eur J Orthod. 2014;36(5):595-602.

27. Okada $\mathrm{M}$, Otsuki $\mathrm{M}$, Tagami J. Effect of nonthermal atmospheric discharge on stain removal of tooth. Dent Mater J. 2019;38(3):396-402.

28. Steiger-Ronay V, Steingruber A, Becker K, Aykut-Yetkiner A, Wiedemeier DB, Attin T. Temperature-dependent erosivity of drinks in a model simulating oral fluid dynamics. J Dent. 2018;70:118-123.

29. Tao D, Smith RN, Zhang $Q$, et al. Tooth whitening evaluation of blue covarine containing toothpastes. J Dent. 2017;67S:S20-S24.

30. Batista GR, Rocha Gomes Torres C, Sener B, Attin T, Wiegand A. Artificial saliva formulations versus human saliva pretreatment in dental erosion experiments. Caries Res. 2016;50(1):78-86.

31. Baumann T, Kozik J, Lussi A, Carvalho TS. Erosion protection conferred by whole human saliva, dialysed saliva, and artificial saliva. Sci Rep. 2016;6:34760. 
Surface roughness $\left(R_{a}\right)$

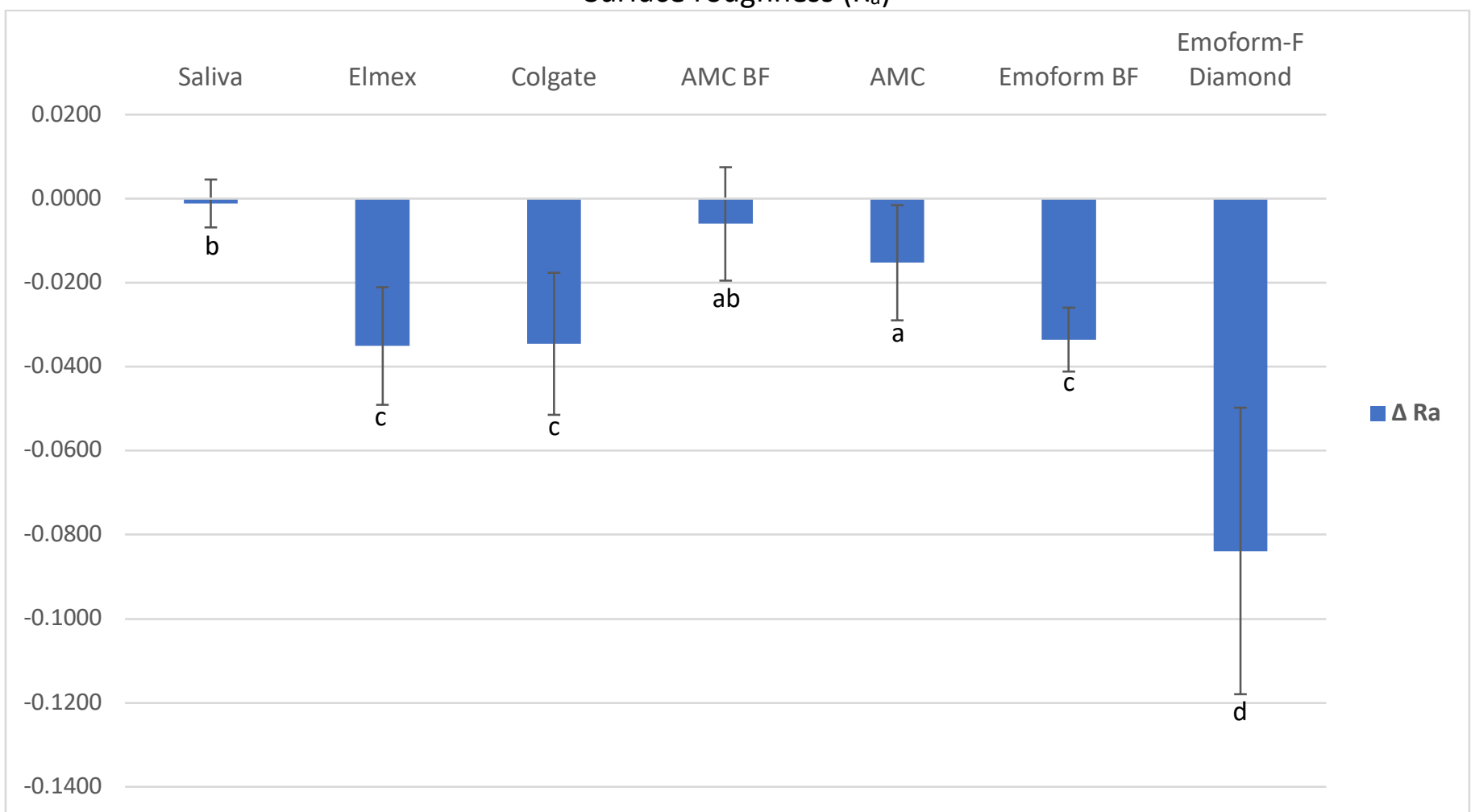

Figure 1: Mean $\Delta R_{a}$ values $(\mu \mathrm{m})$ and standard deviation $(S D)$ after performing the first brushing sequence (values below zero indicate a reduction of roughness). A significant $R_{a}$ improvement was offered in all groups except for artificial saliva and AMC BF. Same letters indicate no significant difference between groups. 
Gloss (GU)

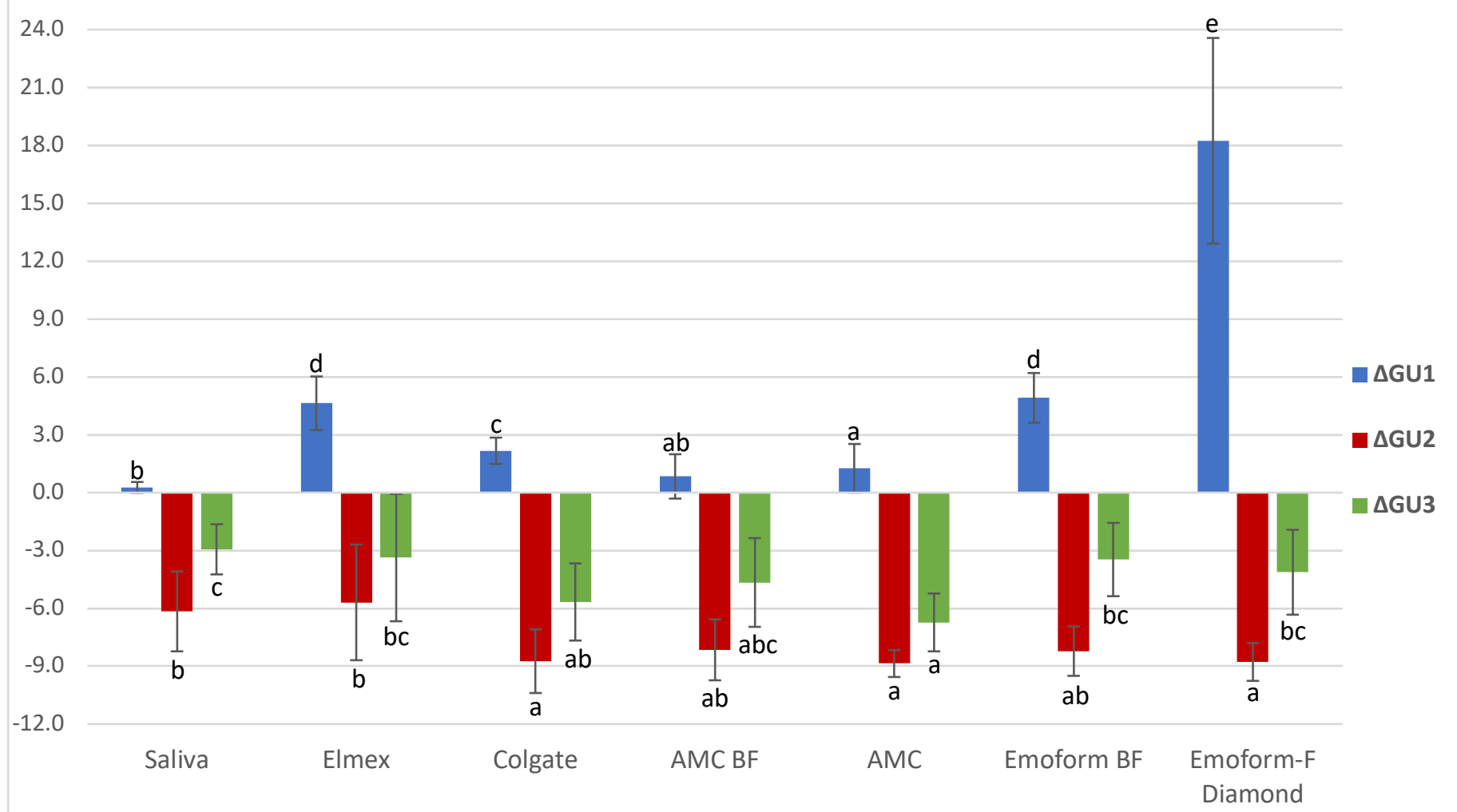

Figure 2: Mean $\Delta G U$ values and SD (positive values indicate an improvement) at each point of observation compared to baseline measurement. $\Delta \mathrm{GU1}=$ after the first brushing sequence, $\Delta \mathrm{GU} 2=$ after the staining protocol, $\triangle \mathrm{GU}=$ after the second brushing sequence. Same letters at each single point of observation indicate no significant difference between groups. Differences within the same group throughout all observation points were always significant. 
Color ( $L^{*}$ values)

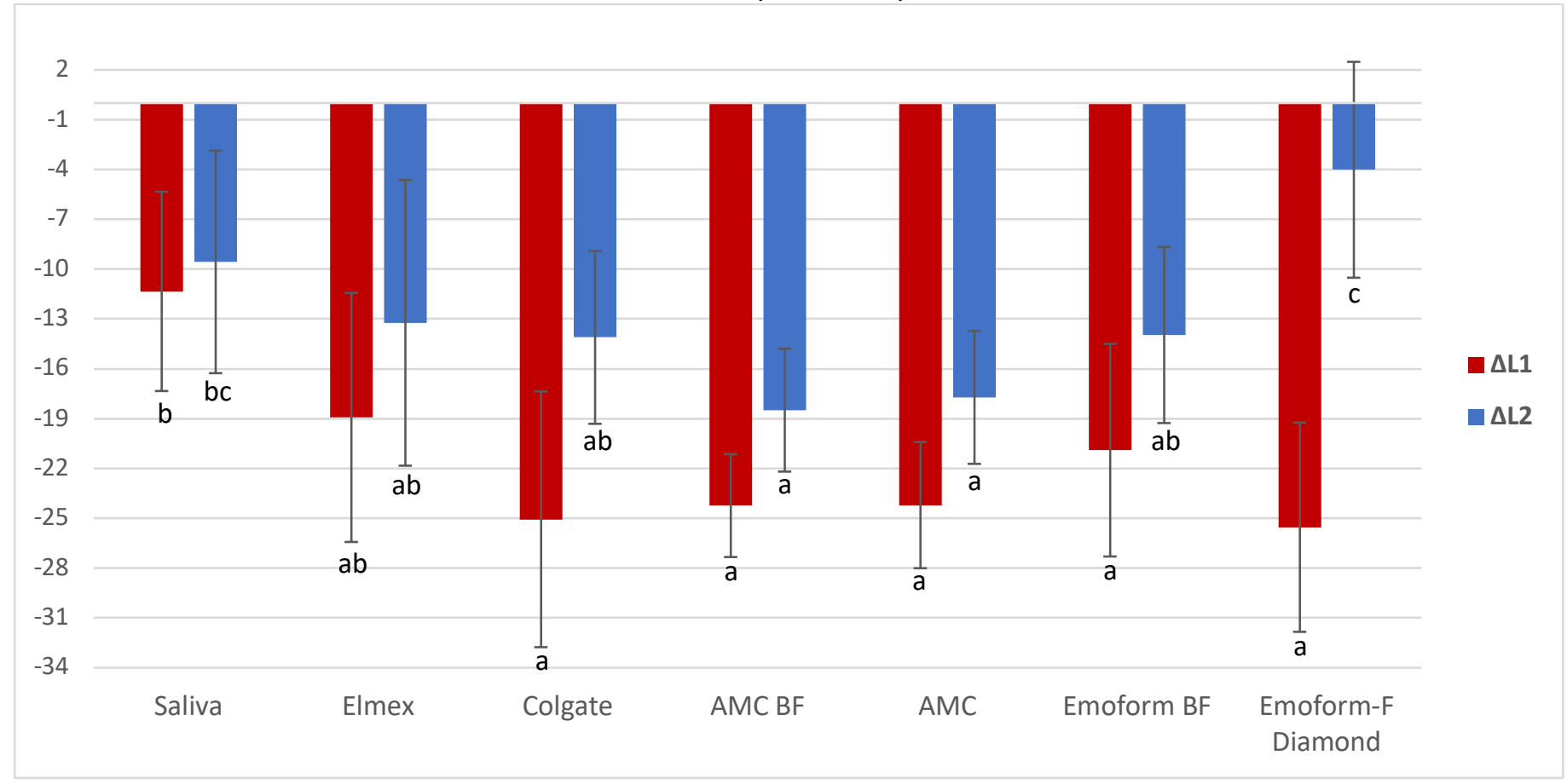

Figure 3: Mean $\Delta \mathrm{L}$ values and SD (positive values indicate an improvement) at each point of observation compared to baseline measurement. $\Delta \mathrm{L} 1=$ after the staining protocol, $\Delta \mathrm{L} 2=$ after the second brushing sequence. Same letters at each single point of observation indicate no significant difference between groups. Differences within the same group throughout both observation points were always significant. 
Color (a* values)

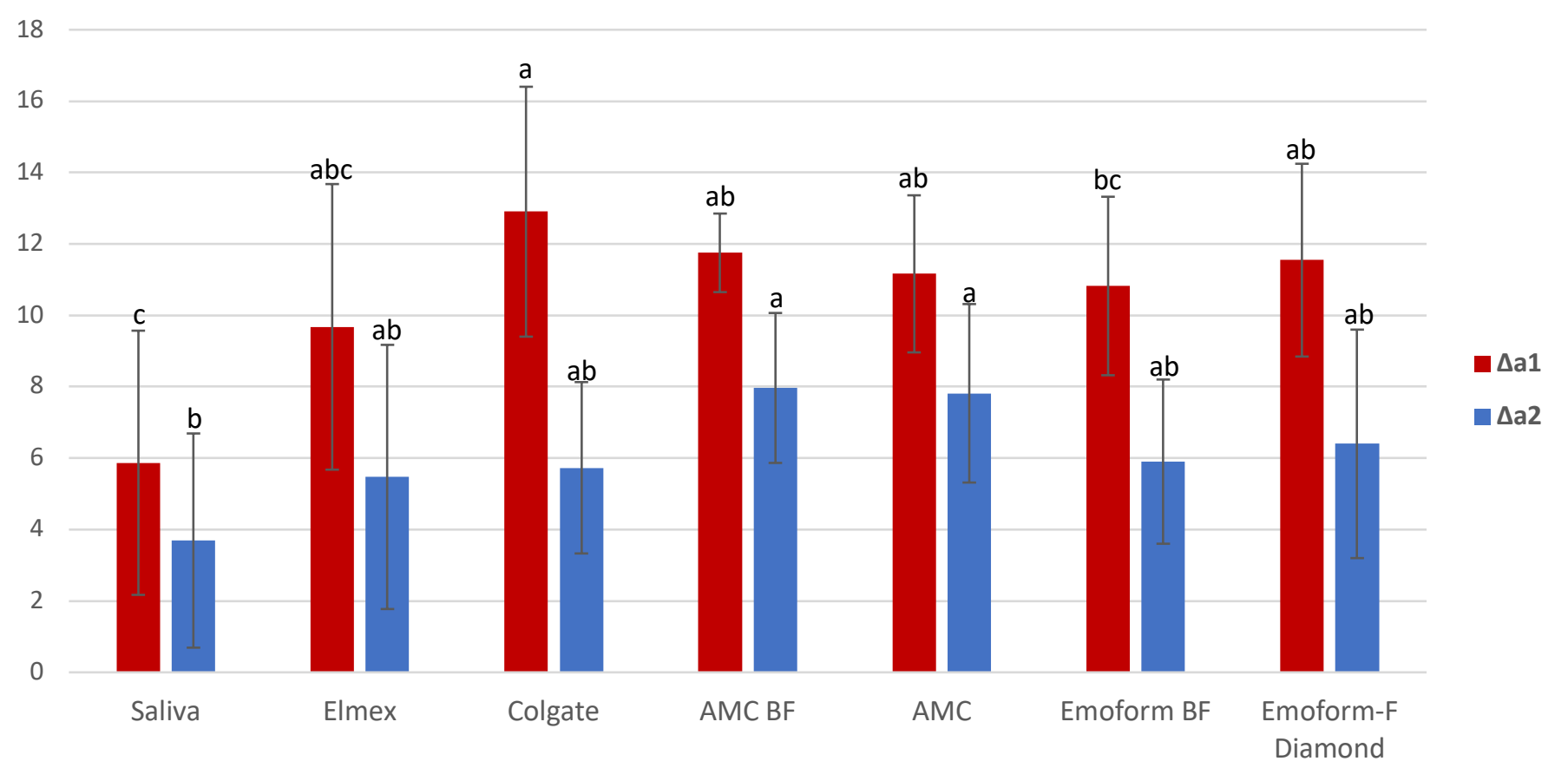

Figure 4: Mean $\Delta$ a values and SD (negative values indicate an improvement) at each point of observation.

$\Delta \mathrm{a} 1=$ after the staining protocol, $\Delta \mathrm{a} 2=$ after the second brushing sequence compared to baseline measurement. Same letters at each single point of observation indicate no significant difference between groups. Differences within the same group throughout both observation points were always significant. 


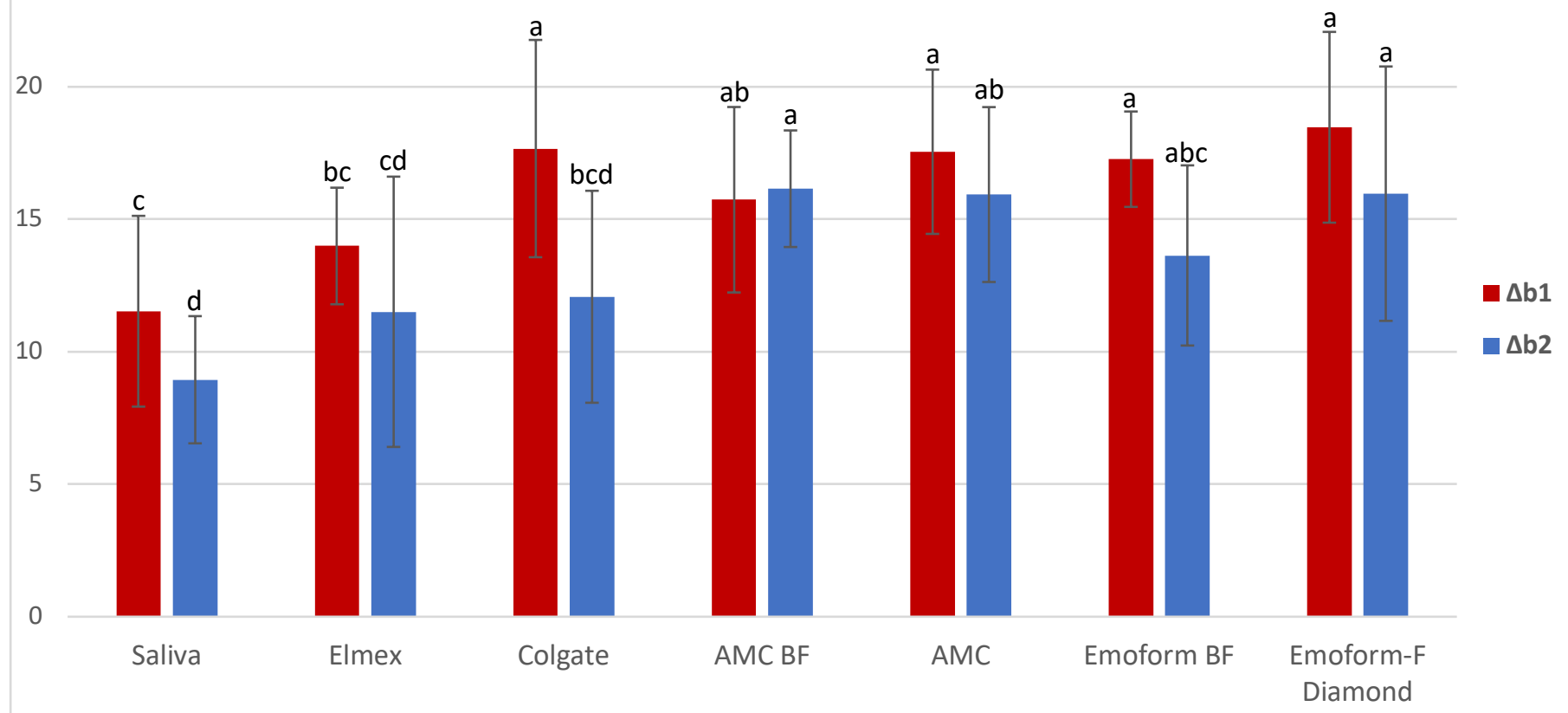

Figure 5: Mean $\Delta \mathrm{b}$ values and SD (negative values indicate an improvement) at each point of observation.

$\Delta \mathrm{b} 1=$ after the staining protocol, $\Delta \mathrm{b} 2=$ after the second brushing sequence compared to baseline measurement. Same letters at each single point of observation indicate no significant difference between groups. Differences within the same group throughout both observation points were significant only for artificial saliva, Colgate Total and Emoform BF. 